\title{
Damage Absorption of High-Impact Structural Systems using Time-Reaction of Hybridized Epoxy-Polyurea Interfaces
}

\author{
By Thomas L. Attard* \\ Hongyu Zhou ${ }^{+}$
}

This study investigates the optimum design in terms of the minimum cost of A carbon-fiber reinforced hybrid-polymeric matrix (CHMC) composite was developed for vibration suppression applications, where the hybrid matrix system was created by combining two polymeric compounds - the epoxy-based phase I which has highly crosslinked morphology and the lightly-crosslinked polyurea elastomeric phase II which when reacted with curing phase I, provides high damping and fracture toughness. The chemical reactions incurring the hybrid matrix system are discussed. The microstructures and micromechanical properties of CHMC are examined through scanning electron microscopy and nanoindentation. Dynamic properties of CHMC as well as conventional carbon-fiber reinforced epoxies are investigated using free vibration and randomly excited vibration tests, and test results indicate CHMC exhibits significantly greater damping than carbon-fiber/epoxy. The influence of two material processing parameters - the polyurea thickness $h p$ and elapsed curing time tc of epoxy - on material damping is investigated. Generally, damping coefficients increase with greater hp and smaller tc.

Keywords: Composite Vibrations, Electron microscopy; Hybrid, Internal friction/damping, Polyurea

\section{Introduction}

Damping is an important property that influences the dynamic behaviors of various materials and structures, in particular the behaviors of those used in vibration sensitive applications. In order to minimize resonance and suppress resonant or near-resonant vibrations, high damping materials are often desired in structural designs. The benefits of using higher-damped structural materials include extending the service life of the various structural components, reducing noise, and reducing structural weight. Most polymeric matrix composites (PMCs) intrinsically have significantly higher damping than metallic materials, and the damping mechanisms in composite materials differ from those observed in conventional structural materials, such as metals and alloys. At the constituent level, the energy dissipation in fibrous composites is

* Associate Professor, University of Alabama at Birmingham, USA.

† Assistant Professor, University of Alabama in Huntsville, USA. 
induced by 1) the viscoelastic/viscoplastic nature of the comprising fiber and matrix constituents, 2) the interaction between material phases, and 3) any damage that may exist in the material, either from a pre-existing condition or newly formed state (Nashif et al., 1985). At the laminate level, the damping of fibrous composites depends not only on the constituent lamina (or ply) properties, but also on the ply orientations and inter-laminar effects (Berthelot and Sefrani, 2003).

In the design of structural materials, it is often contradictory to obtain high stiffness and high damping, i.e., bulk materials that combine high damping and high stiffness are not common (Lakes, 2002). The microstructure of some composite materials gives rise to combined high stiffness and high damping. These materials are often composed of a stiff, low damping phase (e.g. reinforcing fibers) and a compliant, high damping phase. The inclusion of soft particulate phases, such as the core-shell rubber (SCR), into epoxy-based matrices has been proved to be an effective method to improve damping (Cawse and Stanford, 1987; Tsai et al., 2009); however, reduction in material stiffness is often substantial. In this light, multilayered Carbon-fiber reinforced Hybrid-Matrix Composite, or CHMC, is developed to provide high material damping while maintaining ambient stiffness and low fabrication cost. Earlier studies conducted by Zhou and Attard (2013) and Zhou et al. (2013) demonstrated the potential of CHMC as a structural retrofitting material to sustain strength of otherwise brittle substrates and preclude failure. In the present study, chemical reactions involved in CHMC fabrication process are discussed; microstructural and micromechanical properties of CHMC are characterized using scanning electron microscopy (SEM) and nanoindentation; and dynamic properties of CHMC and CHMC/steel composite beams are studied through free vibration and randomly-excited forced vibration tests. Test results are compared to those obtained from carbon-fiber reinforced epoxy (CFE, also commonly referred to as CFRP) beams; and the mechanisms enabling enhanced damping in the CHMC are discussed.

\section{The Carbon-Fiber Reinforced Hybrid Matrix Composite (CHMC)}

\section{Multilayered Morphology, Chemical Composition, and Microstructures}

While the strength and stiffness of continuous fibrous composites are mostly governed by the fiber properties, the matrix phases in composite materials provide stress transferring paths for the reinforcing fibers; thus, the matrix phase, at least to some extent, affects the fracture toughness of the composites (Agarwal et al., 2006) and the general energy dissipation capacities. Most thermosetting polymers, such as epoxies, fracture within a relatively low strain range; as a result, damages initiated in the reinforcing fibers or at the fiber-matrix interface quickly bridge together via matrix cracking causing imminent failure of the material system. One solution to improve the damage tolerance of polymeric composites is to induce "damage barriers" so that microscopic damage events can be isolated, rendering any 
singe crack harmless (Zhou et al. 2013). To implement this concept, elastomeric layers were introduced in the epoxy base matrix. The hybrid polymeric matrix system combines the high stiffness and good fiber saturation of epoxy-based polymers with the high damping and fracture toughness of lightly-crosslinked elastomers.

The multilayered cross-ply morphology of CHMC is schematically shown in Figure 1a and 1b, where the "self-supporting" laminates are stand-alone composite laminates used to manufacture, for example, mechanical components, whereas in a retrofitting application, the laminates may be attached to a substrate, such as steel or concrete, to provide a requisite strength. The hybrid matrix system of the CHMC is created by saturating the reinforcing carbon fiber in an epoxy-based matrix phase I, where the condensation crosslinking reaction between epoxy resin molecules, i.e., in this case the Bisphenol A diglycidal ether, and the hardener (polyetheramine) takes place to form a highly crosslinked structure, see Scheme 1.

Figure 1. Scheme 1: Curing Reaction of Epoxy Resin to Form ThreeDimension Highly Cross-Linked Structure; Scheme 2: The Polymerization Reaction to Form the Polyurea Matrix Phase II; Scheme 3: Epoxy-Polyurea (EP) Time-Sensitive Reaction, Following Elapsed Reaction Time, $\boldsymbol{t}_{c}$, of Scheme 1, Showing Two Plausible Reaction Schemes $(a)$ and $(b)$ of the Major Epoxy and Polyurea Components

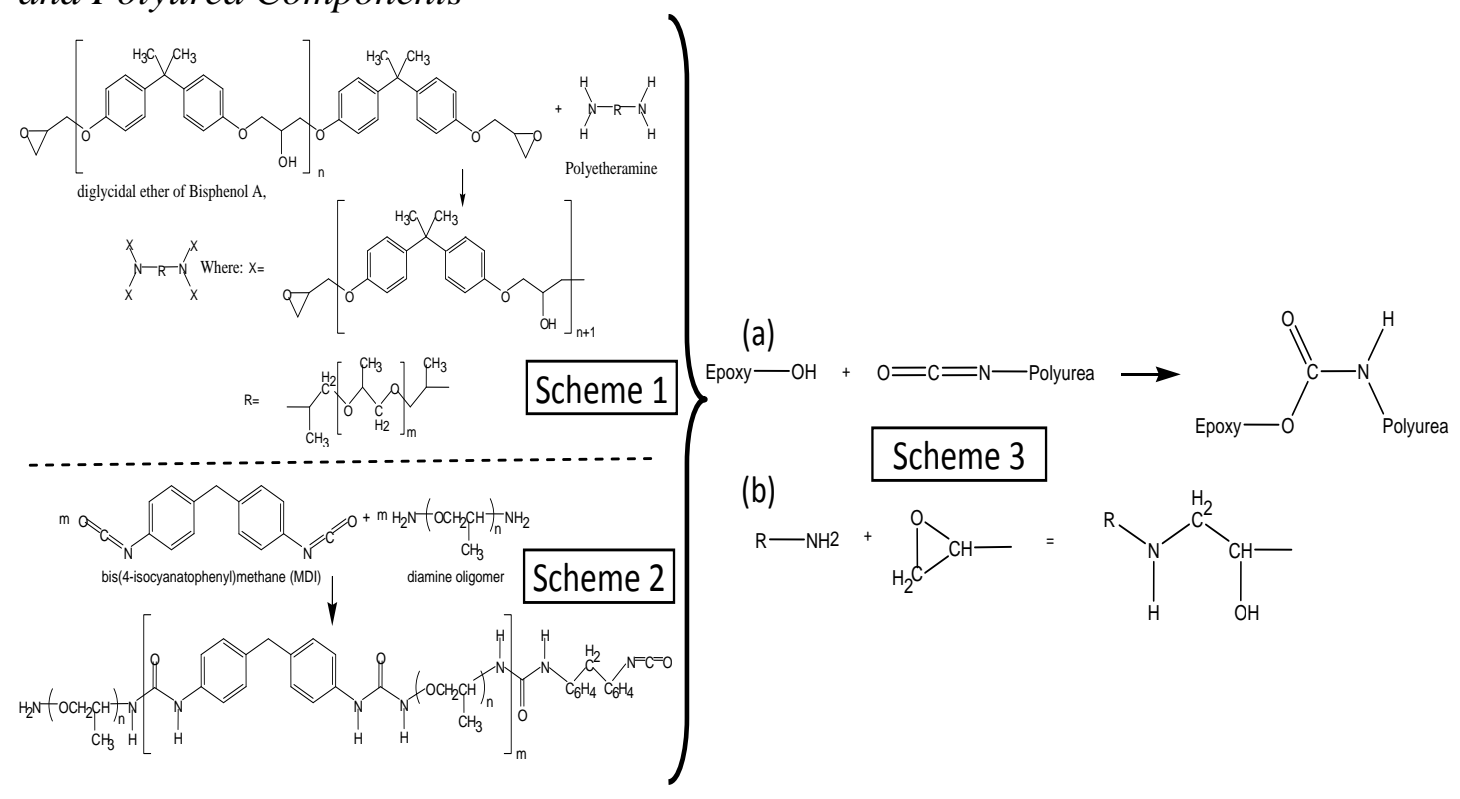

Following a elapsed curing time, $t_{c}$, methylene diphenyl diisocyanate (MDI) and a polyamine hardener are mixed at a molar ratio of (NCO) to $\left(\mathrm{NH}_{2}\right)$ of 1.05 and applied to the surface of the epoxy-based phase I, see Figure 1. The reaction scheme of elastomeric is identified as Scheme 2 to form a lightly crosslinked polyurea elastomer through step-growth polymerization. The thickness of the formed polyurea phase in the matrix system is defined as $h_{p}$, see Figure 1a and 1b. Previous studies (Dai et al., 2009; Kuan et al., 2010) 
reveal two potential kinetic mechanisms of polyurea reacting with a curing epoxy resin. The first is a reaction between the polyurea end group (NCO) and the hydroxide group of the epoxy, see Scheme 3a. A second mechanism may occur through the reactions of amine with epoxide group, see Scheme 3b (Dai et al., 2009). The reacted polyurea-epoxy molecules play a critical role in the CHMC material by: 1) acting as a "compatibilizer" to ensure the interfacial adhesion between the two polymeric matrix phases, which has been shown to be critical for the material to withstand impacts and maintain structural integrity (Zhou et al., 2014); and 2) providing an additional source of internal damping through intermolecular friction or rotational mechanisms (Evans and Ritchie, 1997). Furthermore, studies by Hu et al. (2008), Dai et al. (2009) and Kuan et al. (2010) indicated that the morphology of the polyurea-epoxy reaction product is a function of the reaction time, whereby a transmission electron microscopy (TEM) analysis revealed that with an increase in the reaction time, the width of the polyurea/ epoxy interface increases, leading to higher degree of crosslinking density of the reaction products. As a result, fracture toughness of the polyurea/ epoxy system increased with an increase in reaction time.

Figures $2 \mathrm{~b}-2 \mathrm{e}$ present scanning electron microscopy (SEM) images showing multilayered cross-ply texture of CHMC, where reinforcing carbon fibers are saturated in curing epoxy matrix phase as featured in Scheme 1, and elastomeric polyurea matrix phase II is coated on surface of phase I epoxy. Chemical reaction between epoxy and polyurea phases is evidenced by a "rough" interfacial region, see Figure 2e, where the width is a function of reaction time between polyurea and epoxy resin. The two quantities - the thickness, $h_{p}$, or volume fraction, of the polyurea in the matrix system and the elapsed curing time, $t_{c}$ - as previously defined, are identified as material processing parameters that are later shown to impact certain designable properties of CHMC in performance of structural systems (impact-resistant tornado storm shelters). 
Figure 2. (a) CHMC Configuration for Strengthening a Substrate; (b) SEM Image of CHMC/ Substrate Microstructures; (c) Close-up View of Reinforcing Fiber and Epoxy-Polyurea (EP) System; (d) Reinforcing Carbon Fiber Embedded in Cured Epoxy; (e) Close-up View of EP Interface
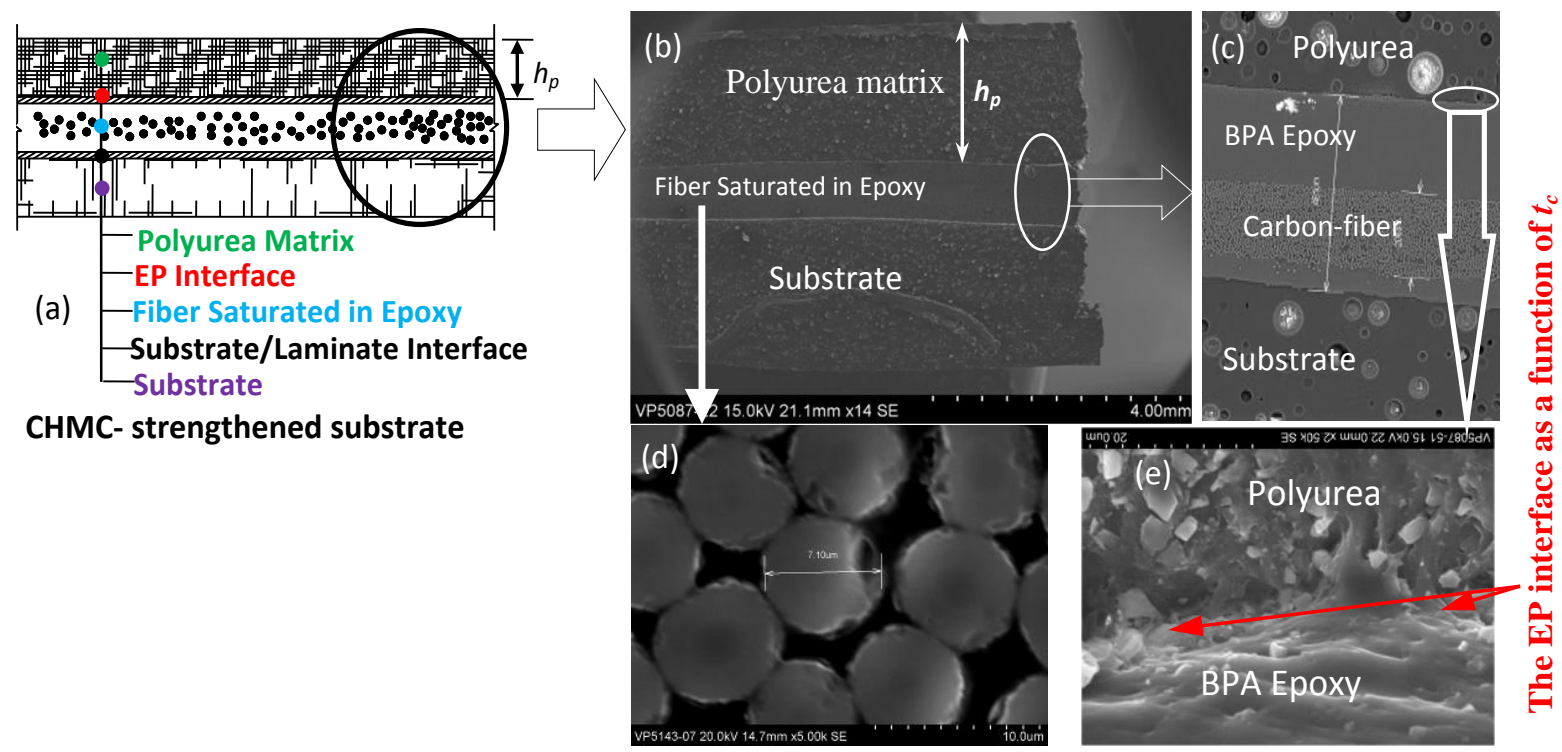

\section{Dynamic Properties, Damping Characterization of CHMC Beams}

\section{Experimental Test Setup and Specimen Configurations}

The experimental equipment that was used to conduct the vibration tests is shown in Figure 3. The specimens are supported horizontally in fixed-fixed condition, and a laser vibrometer was used to measure the velocity responses of one point of the test beams; and two accelerometers were mounted at the midspan and one quarter point to measure the accelerations. The free vibrations of the beams were excited through pulse loading induced by a hammer (applied six times to each specimen). The beam responses were processed using a MatLab code, developed herein, to obtain both the response time-histories and the frequency spectrums for each excitation. A total of 20 beam specimens were designed and fabricated in order to determine the dynamic properties, such as the damping coefficients, of CHMC. The 20 beams were categorized into three groups: 1) four self-supporting beams were tested as stand-alone laminates; 2) six Oberst-type beams, where composite laminates were attached to one side of steel beams, and 3) ten modified Oberst-type beams, where composite laminates were attached symmetrically to both sides of the steel base beam and examined as "retrofitting" cases. The three-types of specimens are shown schematically in Figure 3a. The composite laminates were prepared via a hand lay-up process using TORAY Torayca ${ }^{\circledR}$ unidiractional carbon fiber fabric having a nominal single-ply thickness of $0.33 \mathrm{~mm}$. The epoxy resin was produced by BASF. The properties reported by the manufacturers can be found 
from the manufacture's data sheets (BASF CF 130 Guide, 2007; BASF Primer Guide, 2007).

Figure 3. (a) Illustrative Figure Showing Test Setup and Specimen Configurations; (b) Test Set-up and Instrumentation; and (c) Close-up View of Test Specimen

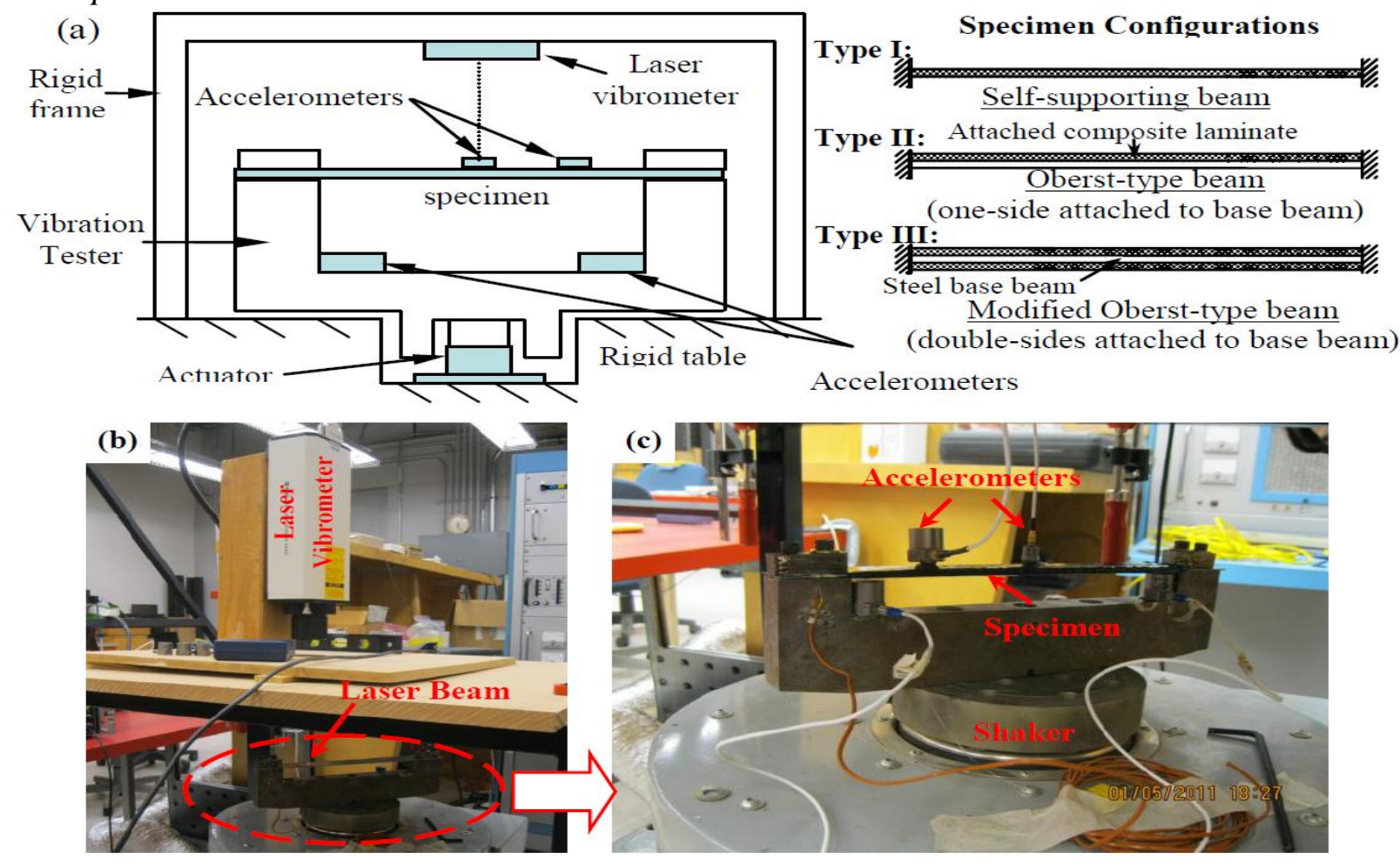

The forced vibration tests were performed using a shaker, as shown in Figures $3 \mathrm{a}$ and $3 \mathrm{~b}$. Random excitation signals, i.e., the banded white-noise having peak acceleration amplitudes of $0.3 \mathrm{~g}$ and $3 \mathrm{~g}$, were used to determine the frequency-dependent properties of the beams. Two additional accelerometers were mounted on the shaker base to ensure that the actual excitation signal complied with the desired input signal.

\section{Experimental Analysis Method}

Self-Supporting Beams

The governing equation of an un-damped uniform Euler-Bernoulli beam in transverse free vibration may be written in the form of equation (1) as

$$
\frac{E I}{\rho A} \frac{\partial^{4} w}{\partial x^{4}}(x, t)+\frac{\partial^{2} w}{\partial t^{2}}(x, t)=0
$$

where $E$ is the Young's Modulus of the beam material, $A$ and $I$ are the area and moment of inertia of the beam cross-section, respectively; $\rho$ is the mass density;; $w(x, t)$ is the transverse deflection of the beam as a function of the 
location $x$ along the beam, and time $t$; (Rao, 2007). The solution of equation (1) can be obtained by assuming the transverse displacement of the beam to be

$$
w(x, t)=W(x) T(t)
$$

then equation (1) becomes

$$
\frac{E I}{\rho A} \frac{1}{W(x)} \frac{d^{4} W(x)}{d x^{4}}+\frac{1}{T(t)} \frac{d^{2} T(t)}{d t^{2}}=\omega^{2}
$$

Or

$$
\begin{aligned}
& \frac{\partial^{4} W(x)}{\partial x^{4}}-\frac{\rho A}{E I} \omega^{4} W(x)=0 \\
& \frac{\partial^{2} T(t)}{\partial t^{2}}+\omega^{2} T(t)=0
\end{aligned}
$$

where $\omega$ is the natural frequency. The solution of equation (6) may then be expressed as

$$
\begin{aligned}
W(x)= & C_{1}[\cosh (\beta x)+\cos (\beta x)]+C_{2}[\cosh (\beta x)-\cos (\beta x)] \\
& +C_{3}[\sinh (\beta x)+\sin (\beta x)]+C_{4}[\sinh (\beta x)-\sin (\beta x)]
\end{aligned}
$$

where

$$
\beta^{4}=\frac{\rho A}{E I} \omega^{2}
$$

and $A, B, C_{1} \sim C_{4}$, are constants that may be determined by the imposing boundary conditions. For lightly damped self-supporting uniform beams fixed at both ends, see Figure 3a - type I, boundary conditions $w(0)=0, \frac{\partial w}{\partial x}(0)=0, w(L)=0, \frac{\partial w}{\partial x}(L)=0$ may be substituted into equation (6), leading to

$\cos (\beta L) \cosh (\beta L)=1$

The approximate solution of equation (10) may be expressed as

$$
\beta_{i} L=(2 i+1) \pi / 2, i=1,2,3 \ldots
$$

Recalling equation (7), the natural frequency of mode $i$ may be calculated as 


$$
\omega_{i}=2 \pi f_{i}=\frac{4}{(2 i+1)^{2} \pi^{2}} \sqrt{\frac{E I}{\rho A L^{4}}}, i=1,2,3 \ldots
$$

where $f_{i}$ is natural frequency of mode $i$ in $\mathrm{Hz}$. For a viscoelastic beam subjected to harmonic loading of frequency $\omega$, elastic moduli of the materials may be expressed as complex quantities

$$
E_{\omega}^{*}=E_{\omega}^{\prime}\left(1+i \eta_{\omega}\right)
$$

where $E_{\omega}^{*}$ is the complex Young's modulus of the beam material at frequency $\omega ; \eta_{\omega}$ is the loss factor associated to the dynamic Young's modulus (or storage moduli) $E_{\omega}^{\prime}$. Thus, the storage modulus of the beam material, $E_{\omega}^{\prime}$, may be expressed as a function of $\omega_{i}$, via equation (12)

$$
E_{\omega}^{\prime}=\frac{\omega_{i}^{2} L^{4}}{[(2 i+1) \pi]^{4}} \frac{\rho A}{I}
$$

The loss modulus, $E_{\omega}{ }^{\prime \prime}$, is then calculated using the storage modulus and the composite damping coefficient (or the loss factor) via equation (13) as

$$
E_{\omega}^{\prime \prime}=E_{\omega}^{\prime} \tan \eta_{i} \approx 2 E_{\omega}^{\prime} \xi_{i}
$$

where $\eta_{i}$ and $\xi_{i}$ are the loss factor and damping coefficient of mode $i$, respectively.

Experimental measurements of the damping coefficient, $\xi$, or the loss factor, $\eta$, may be evaluated using logarithm decrement method (when analyzing free response data in time domain) via equation (14)

$$
\xi=\left[\frac{1}{\omega_{1}(n-1) T_{d}}\right] \ln \frac{x_{1}}{x_{n}}
$$

where $x_{1}$ are $x_{n}$ are the amplitudes of the first and $n^{\text {th }}$ vibration cycles that are used for the logarithm decrement calculation, $\omega_{1}$ is the natural frequency of first mode vibration, expressed in $\mathrm{rad} / \mathrm{sec}$, and $T_{\mathrm{d}}$ is the period of vibration of the first cycle of the damped response; or the half-power bandwidth method (when analyzing response in the frequency domain) via equation (15)

$$
\xi_{i}=\frac{\Delta f_{i}}{2 f_{0, i}}
$$

where $f_{0, i}$ is the resonance frequency of mode $i$, in $\mathrm{Hz}$, and $\Delta f=f_{2}-f_{1}$ is the frequency difference between the half-power points, i.e. frequencies at which 
the response equals the one at resonance divided by $\sqrt{2}$, as presented in Figure 4a.

Figure 4. Velocity Spectrum and PSD Obtained via: (Free Vibration Test; and (b) Forced Random Excitation Test

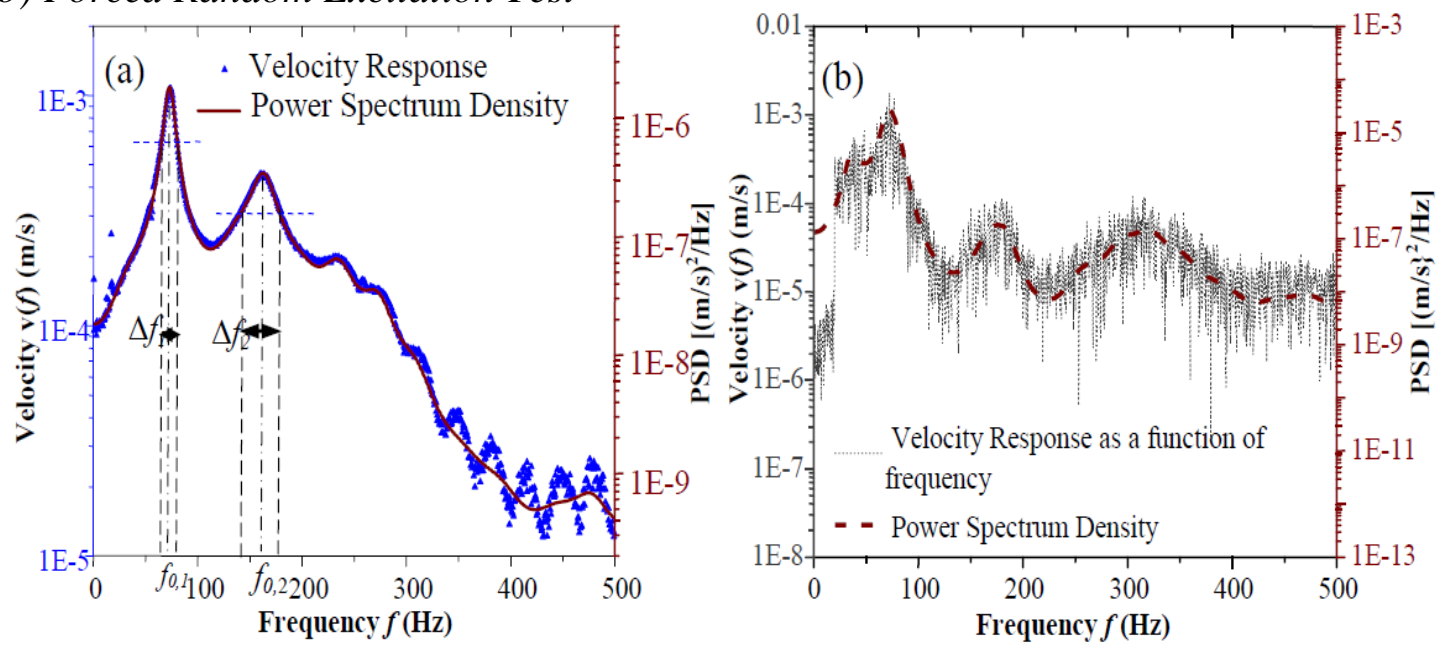

The dynamic characteristics and damping properties of the composite beams may also be determined using forced vibration, where a limited bandwidth white noise excitation signal is input into the system. The corresponding response is recorded by a laser vibro-meter or using accelerometers. The measured time-history responses were converted into frequency spectrums via Fast Fourier Transformation (FFT), and the power spectral density (PSD) distributions, which quantify the power in the response per unit frequency, for each forced vibration test were estimated using the "pyulear" tool box in MatLab based on the Yule-Walker autoregressive method (Gersch and Sharpe, 1973), see Figures 4a and 4b. The damping coefficients, or loss factors, are determined via the half-power bandwidth method as expressed by equation (16). Note that the half-power point corresponds to a drop by $50 \%$ of the power in comparison of the value at the neighboring peak.

\section{Beams One-side Attached with the Composite Laminate, Figure 2a}

The analysis of the Oberst-type beams, i.e., steel base beam with the composites laminate attached on one side as shown in Figures $2 \mathrm{a}$ and $3 \mathrm{a}$ - type II, follows the classical Euler-Bernoulli beam theory, where the rotational inertia and shear deformation were neglected (Nashif and Jones, 1985; ASTM, 2010). The Young's modulus of the base beam and composite laminate are denoted as $E_{b}$ and $E$, respectively; and the thickness of the base beam and the laminate are $H_{b}$ and $H$; the resonant frequencies (in $\mathrm{Hz}$ ) of the composite beam (base beam attached with composite laminates) and the laminate are $f_{c}$ and $f_{n}$; and if the loss factors of the composite beam and the laminates are denoted as $\eta_{c}$ and $\eta$, respectively, then the elastic modulus and loss factor of the laminate may be calculated using equations (16) and (17) as 


$$
\begin{aligned}
& E=\frac{E_{b}}{2 T^{3}}\left[(\alpha-\beta)+\sqrt{(\alpha-\beta)^{2}-4 T^{2}(1-\alpha)}\right] \\
& \eta=2 \xi=\eta_{c}\left[\frac{(1+M T)\left(1+4 M T+6 M T^{2}+4 M T^{3}+M^{2} T^{4}\right)}{M T\left(3+6 T+4 T^{2} 2 M T^{3}+M^{2} T^{4}\right)}\right]
\end{aligned}
$$

where $M=E / E_{b}$ and $T=H / H_{b}$ are the Young's modulus and thickness ratios of the laminate and base beam materials, respectively. And

$$
\begin{aligned}
& \alpha=\left(\frac{f_{c}}{f_{n}}\right)^{2}\left(1+D_{\rho} T\right) \\
& \beta=4+6 T+4 T^{2}
\end{aligned}
$$

where $D_{\rho}=\rho / \rho_{b}$ is the density ratio of the laminate and the base beam material [1].

\section{Results and Discussion}

Self-Supporting Beams

Free vibration time-history responses of steel, the carbon fiber reinforced epoxy, and the type-I CHMC $\left(t_{\mathrm{c}}=2.5 \mathrm{hr}\right.$.) self-supported beams with similar initial conditions are plotted in Figure 5a. It is obvious from the time-history responses that damping of the CHMC beams is substantially higher than that of carbon fiber/ epoxy and steel beams. And the first-mode damping coefficients for the five types of the self-supporting beams, i.e., steel, carbon fiber reinforced epoxy, and three types of the CHMC beams having various $t_{c}$, are compared in Figure 5b. According to Figure 5b, the damping coefficients of the self-supported beams decrease as the intermittent curing time $-t_{c}$ - increases. This may be explained by the fact that a portion of the damping component had originated from the polymeric interfacial region as shown in Figure 1e and featured by the reaction Scheme 3, where the molecular structure is affected by the intermittent curing time $t_{c}$. Figure $5 \mathrm{c}$ and $5 \mathrm{~d}$ present the different sizes and morphologies of the polymeric interfacial EP phase regions, resulting from various $t_{c}$ values, i.e. $t_{c}=2.5 \mathrm{hr}$. versus $t_{c}=3.5 \mathrm{hr}$. A comparison of the interfaces shows that the former has a 'rougher' pattern due to a lower $t_{\mathrm{c}}$, i.e., longer reaction time between polyurea and epoxy. As discussed earlier, $t_{c}$ was defined as the curing time of the epoxy matrix phase I (Scheme 1) before application of polyurea prepolymers, where lower $t_{c}$ may result in lower crosslinking density at EP interfacial region as curing reaction of epoxy molecules is "interrupted" by the polyurea-epoxy reactions (Scheme 3) at an intermittent stage, thus altering the curing kinetics. Thus, the wider and more "jagged" EP interface possibly indicates a more compliant interfacial covalent molecular bond of the hybridized polymer structure, resulting in higher mobility of the polymeric molecular chain, consequently enabling internal 
friction between the molecules to produce higher damping and a larger molecular rotation component to exist (Evans and Ritchie, 1997).

Figure 5. (a) Velocity Time-Histories (under Free Vibration) of Steel, CarbonFiber Reinforced Epoxy, and CHMC Beams; (b) Damping Coefficient Calculated using Free Vibration Responses; and (c) and (d) EP Interface Morphologies for $\boldsymbol{t}_{c}=2.5 \mathrm{hrs}$ and $\boldsymbol{t}_{c}=3.5 \mathrm{hrs}$, Respectively
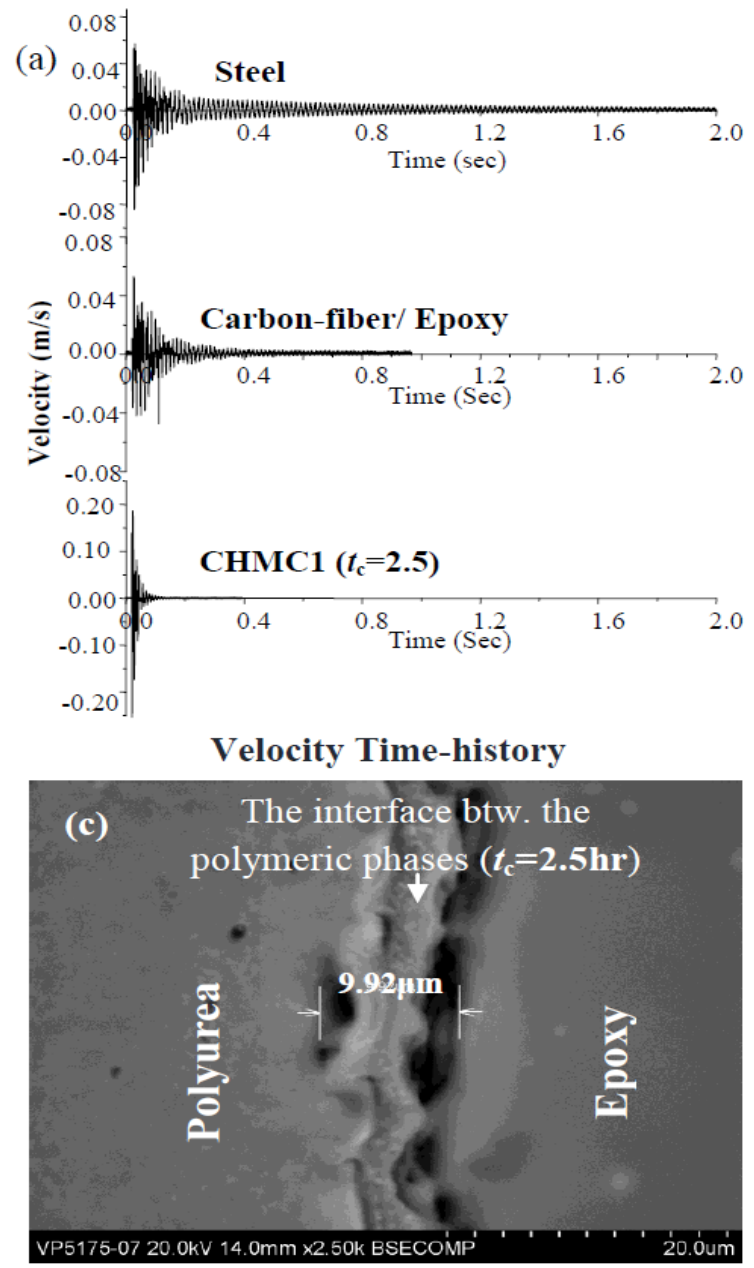
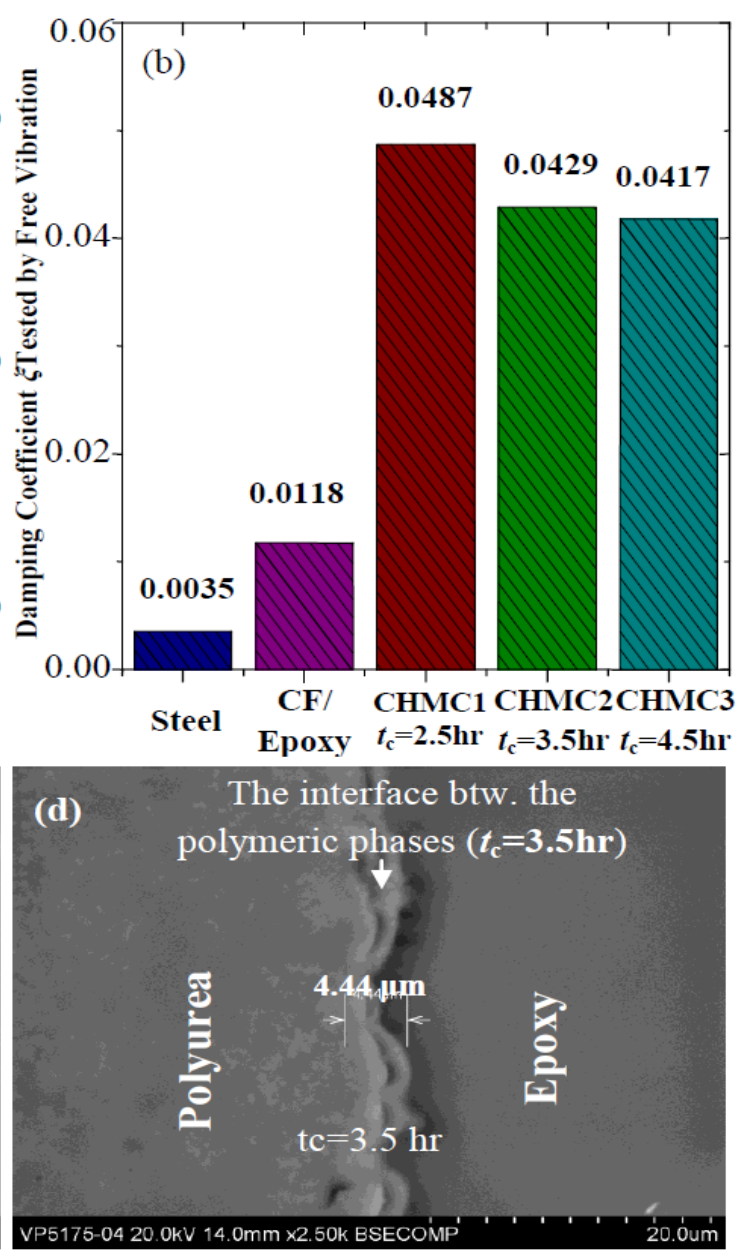

Forced vibration responses of the self-supported beams are obtained by exciting specimens by band-limited white noise. Two peak excitation accelerations (PEAs), i.e., 0.3g and 3g, were used to excite the beams, and velocity responses are measured using a laser vibrometer. Power spectral density (PSD) of self-supporting beams made from steel, carbon fiber reinforced epoxy, and type-I CHMC $\left(t_{\mathrm{c}}=2.5 \mathrm{hr}\right.$.) are shown in Figures 6a and $6 \mathrm{~b}$ for PEA equal to $0.3 \mathrm{~g}$ and $3 \mathrm{~g}$, respectively. It is evident that, under the same excitation condition, energy content of the velocity response signal of CHMC beam is significantly lower compared to the energy in steel and carbon fiber reinforced epoxy beams, especially at higher modes of vibration. The increased suppression of higher modes of vibration is critical for applications that are sensitive to higher frequency vibrations, such as equipment housing (Ungar 
and Dietrich, 1966). Modal damping coefficients calculated by the half-power bandwidth method, equation (15), for the first three vibration modes of CFE and three types of CHMC beams are plotted in Figure 6c. Dependency of modal damping coefficients to intermittent curing time $-t_{c}$ - for the first three vibration modes generally follows the same trend as the free vibration test results, i.e., damping coefficients decrease as $t_{c}$ increases, and modal damping coefficients, in general, appear to slightly increase at higher frequencies. This is consistent with observation of the PSD relationships in Figures $6 \mathrm{a}$ and $6 \mathrm{~b}$, showing that the spectrum peaks of higher modes of vibration had "flattened" due to higher damping in the higher frequency range. Storage and loss moduli of CF/epoxy and CHMC calculated per equations (12) and (13) are compared in Figure 6d.

Figure 6. Forced Vibration Test Results of Self-Supporting Beams: (a) PSD Obtained for Steel, Carbon Fiber/ Epoxy, and CHMC Beams (PEA = 0.3g); $(b)$ PSD Obtained for Steel, Carbon Fiber/ Epoxy, and CHMC Beams $(P E A=3 g)$; (c) Damping Coefficient; (d) Storage and Loss Moduli
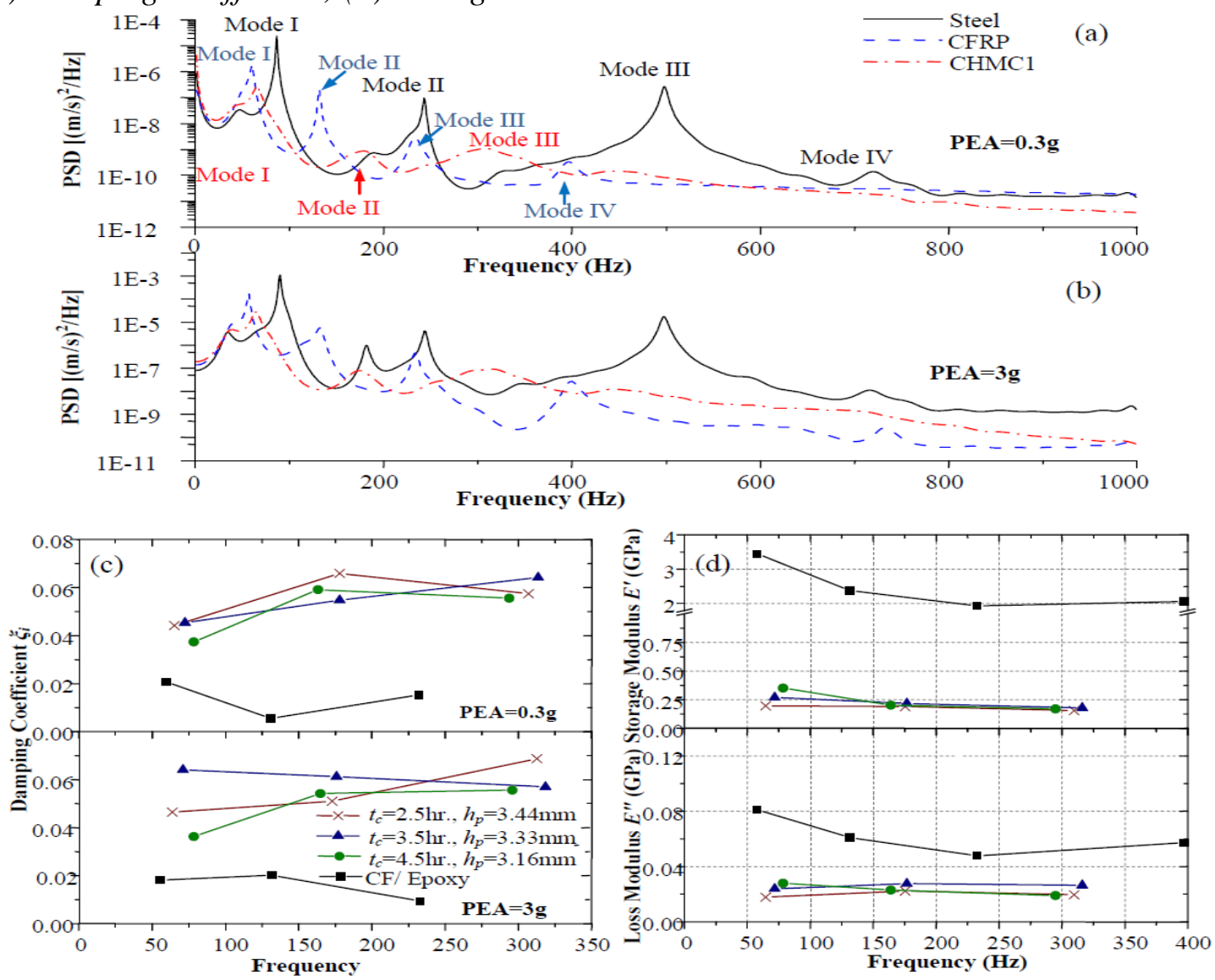

Beams Having One-Side Attached to the Composite Laminate

When materials are used as retrofitting systems for a substrate, such as steel or concrete, they are often applied as thin laminates or coatings. It is often not feasible to test the dynamic properties of the retrofitting material system as 
a self-supported system. In such cases, the retrofitting material can be attached to one side (Oberst-type) or both sides (modified Oberst-type) of a base beam, as shown Figure 3. The properties of the retrofitting laminate, such as the smeared elastic modulus and damping coefficient (or loss factor), can be determined from the dynamic responses of the composite beam and the base beam according to equations (16)-(21).

The calculated damping coefficient of the retrofitting laminate, $\xi$, based on equation (17) for the Oberst-type beams are plotted in Figure 7a as functions of frequency. Damping coefficients of the CHMC used as retrofitting laminates are shown lower than those of self-supporting laminates due to the smaller volume fraction of the high damping constituent - the polyurea matrix phase II, since the elastomer can only be applied to one side of the retrofitting laminate. Comparing the first mode damping coefficients of laminates having the same $t_{c}$, one can find that the damping coefficient is higher with a greater thickness of the matrix phase II, i.e., higher $h_{p}$. As mentioned in the previous sections, the $t_{c}$ affects the morphology of the polyurea-epoxy interface, and in turn has an impact on the damping the cohesion between this two matrix phases. The differing interface morphologies have been demonstrated critical for impact resistance of the composite (Zhou et al., 2014). The dependence of frequency on the damping coefficients of the CHMC-retrofitted beams follows the same trend as the self-supporting laminates, i.e., damping increases as the driving frequency increases. This observation may be a result of the viscous properties of the composite constituents, particularly polyurea. The storage and loss moduli of the attached composite laminate may be calculated using the forced vibration responses based on equations (12) and (13), respectively. The results are plotted in Figure $7 \mathrm{~b}$.

Figure 7. Dynamic Properties of CHMC Obtained from Oberst Beam: (a) Damping Coefficiennts; (b) Storage and Loss Moduli as Functions of Frequency

The Oberst-type beams (single side attached with CHMC):
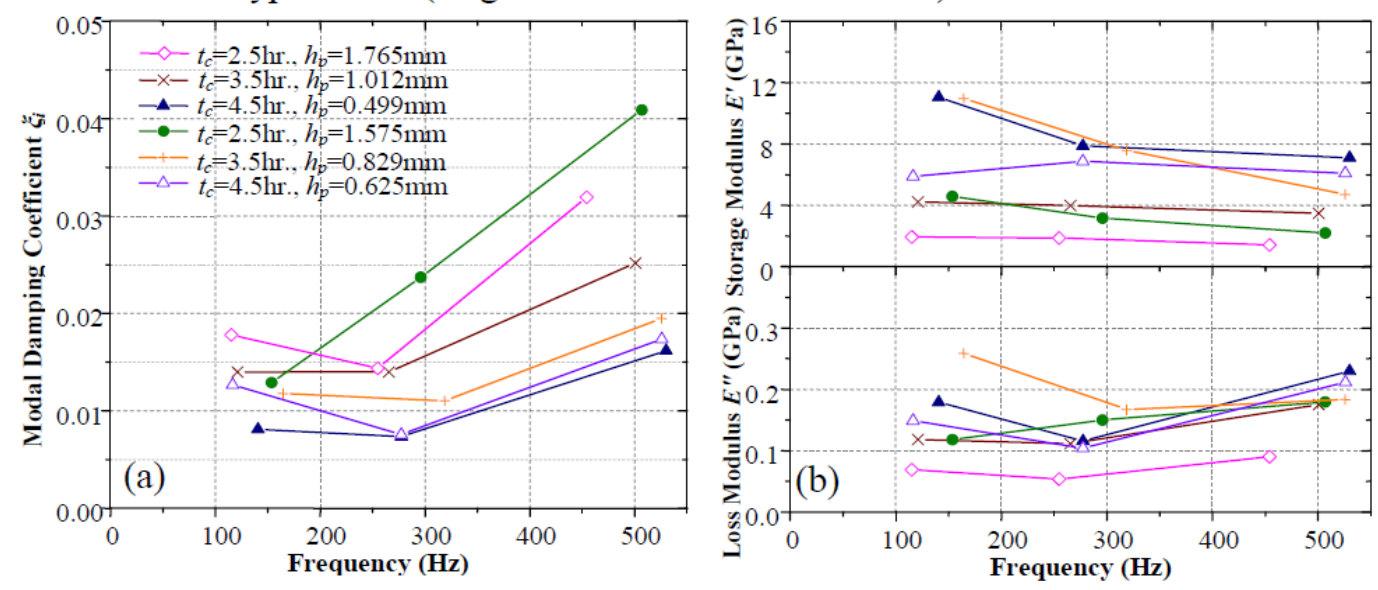


\section{Applications to Wind-Borne Debris Impact Tests}

In an application to tornado safe-room design, a series of tests was performed on wall components of above-ground tornado storm shelters (Zhou et al., 2014). Seven wall panels were designed with CHMC and subjected to EF-4 and EF-5 level wind-borne projectiles at the National Wind Institute (NWI) at Texas Tech University. Windborne debris was projected at $90 \mathrm{mph}$ (EF-4, $200 \mathrm{mph}$ ground wind speed tornados) and $100 \mathrm{mph}$ (EF5, $250 \mathrm{mph}$ ) in accordance with FEMA 320, 361, and ICC500 (see reference), Figures 8a and 8b. A comparison between the performance of CHMC-designed panels and carbon fiber/ epoxy (CFE)-designed panels was made. Each wall used the same amount of carbon fiber and layup procedures, except that polyurea was not applied to the CFE-panels. Using $2 \times 4$ missiles to replicate EF-4 and EF-5 impacts in accordance to FEMA 320, 361, and ICC500, the CFE-designed panels were fully penetrated and failed (Figure 8c). However, CHMC-designed panels (Specimen 3), having an EP interface that was manufactured by applying a polyurea coating $3 \mathrm{~mm}$ to an epoxy that had been curing for $2.5 \mathrm{hrs}$, passed the EF-5 impacts (Specimen \#3 in blue, Figure 9a). Specimen \#2 (in red, Figure 9a), however, was manufactured by applying a polyurea coating $\underline{5 \mathrm{~mm}}$ thick to an epoxy that had been curing for $3.5 \mathrm{hrs}$. Specimen 2 failed its EF-5 impact but passed the EF-4 impact (Figure 5b), implying a correlation between the material/ mechanical properties and $t_{c}$, which appears to control the "roughness" and width of the EP interface (Figures 5c and 5d). One explanation for the trends in Figure 9a (blue vs red) may be that a rougher interface enables greater interfacial molecular mobility, resulting in enhanced kinetic impact energy dissipation, inferring that kinetic energy dissipation is a function of $t_{c}$, where quantity of polyurea has less influence on energy dissipation at higher load rates. 
Figure 8. Tornado Impact Testing of Storm Walls: (a) Air Cannon Set-up at the NWI; (b) Impacted Storm Wall, Comprised of a Thin (White) CHMC Panel (6-8mm) and Support Structure $(3 / 4$ in \& $3 / 8$ in Plywood, $2 x 4 s$, Nails); and (c) Complete Failure of a CFE (Carbon-Fiber Reinforced Epoxy, same as CFRP) Panel and Wall System, with Full Missile Penetration

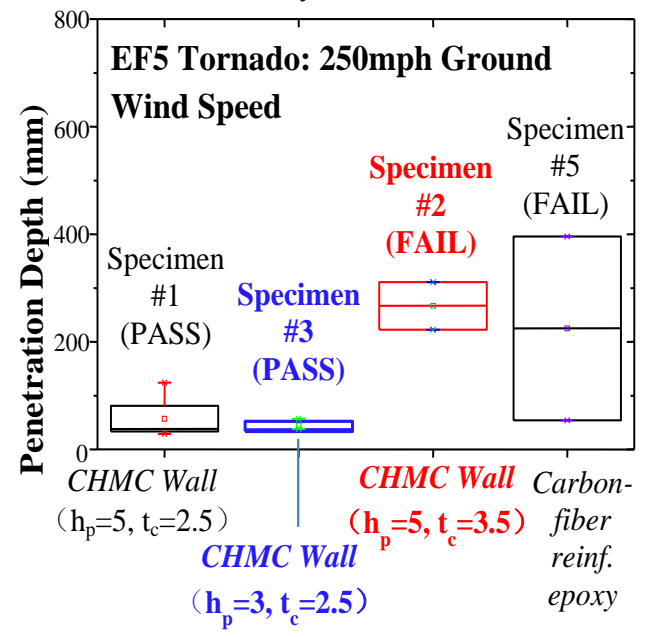

(a)

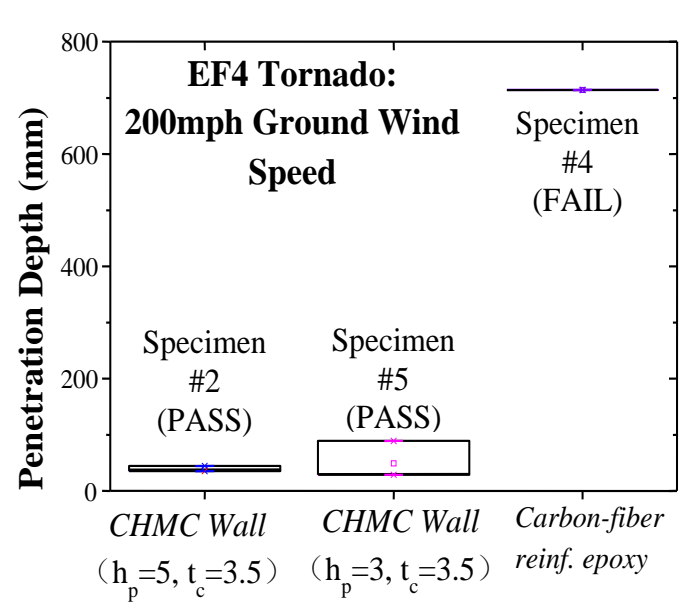

(b)

Figure 9. CHMC Wall Panels Subjected to (a) EF-5 and (b) EF-4 Windborne Testing

(a)
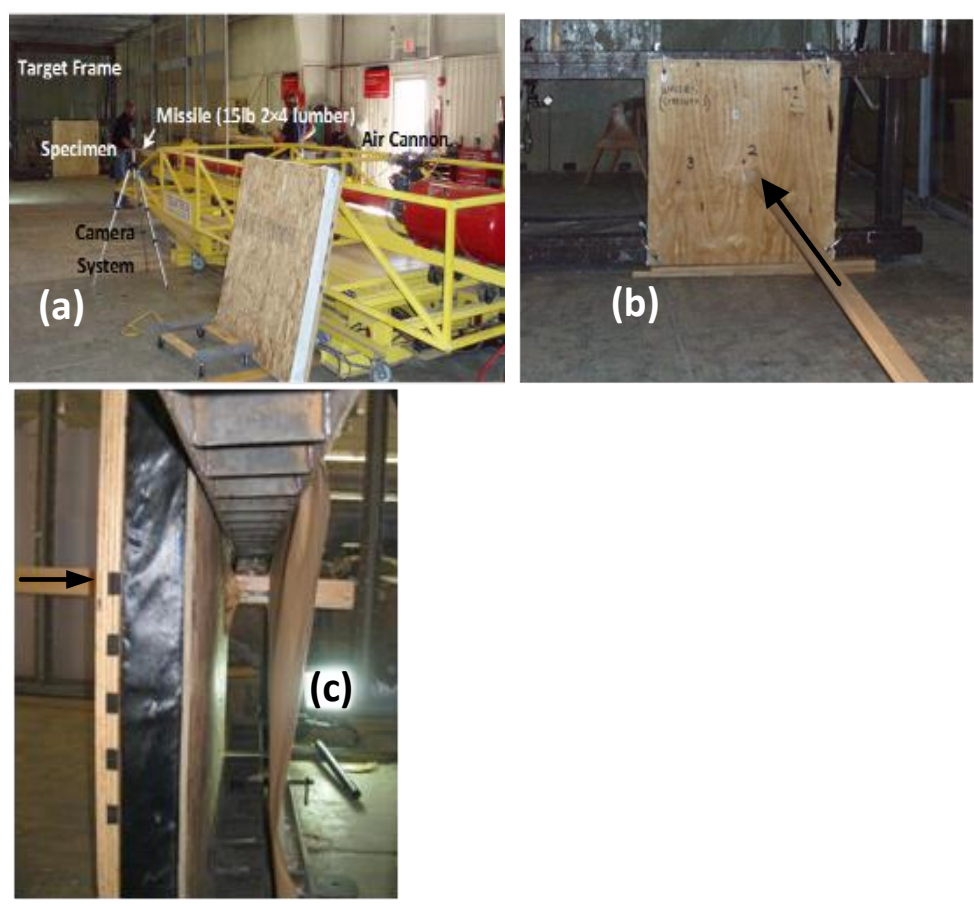


\section{Conclusions}

A carbon-fiber reinforced polyurea-epoxy hybrid matrix composite was developed for vibration suppression applications. Scanning electron microscopy (SEM) images showing microstructures of the CHMC are presented. A multilayered cross-ply texture was evidenced by SEM images, and micromechanical properties of the constituents were studied using nanoindentation tests; the cross-ply modulus profile of the CHMC laminate was obtained, and nanoindentation results indicate substantial hysteretic damping properties of the matrix constituents, where crosslinking reaction of the epoxy phase was altered by adding polyurea phase. Reaction between epoxy and polyurea (EP) phases formed a well-bonded interface, which is believed to provide substantial additional internal damping for the CHMC.

Dynamic properties of the CHMC and conventional carbon fiber reinforced epoxies ( $\mathrm{CF}$ / epoxy) are investigated using free vibration and forced vibration tests. Natural frequencies and damping coefficients were calculated using vibration responses of tested beams. CHMC exhibits enhanced damping and vibration suppression capability than the conventional $\mathrm{CF} /$ epoxy both as a stand-alone structural material and as a retrofitting system. In general, the observed damping is higher when used as a stand-alone laminate than as a retrofitting material. This may be attributed to the single-side coated lay-up of the retrofitting laminates, thus, the lower volume fraction of the polyurea phase. The influence of the two material processing parameters $-h_{p}$ and $t_{c}$ - on the material damping was investigated, and the results reveal that, generally, the damping coefficients increase with greater $h_{p}$ and smaller $t_{c}$. Damping based design of CHMC is facilitated through material processing parameters, including matrix phase II thickness - $h_{p}$ - and intermittent curing time - $t_{c}$, which impact its material properties.

\section{Acknowledgments}

This research was partially supported by Department of Homeland Security through the Higher Education Research Experience (HERE) Program, and by the Southeast Region Research Initiative (SERRI) at the Department of Energy's Oak Ridge National Laboratory (ORNL), DHS Project No. 90300.

\section{References}

Agarwal B., Broutman L. J. and Chandrashekhara K. Analysis and performance of fiber composites. $3^{\text {rd }}$ Edition ed. New York, NY: Jon Wiley \& Sons; 2006.

ASTM E756-05. Standard test method for measuring vibration-damping properties of materials. West Conshohocken, PA: ASTM; 2010.

BASF Mbrance CF 130 Data Guide. Shakopee, MN: BASF Construction Chemicals, LLC - Building Systems; 2007. 
BASF Mbrace Primer Data Guide. Shakopee, MN: BASF, The Chemical Company; 2007.

Berthelot J. M. and Sefrani Y. Damping analysis of unidirectional glass and Kevlar fibre composites. Comp Sci \& Tech 2003;64:1261-78.

Cawse J. L., Stanford J. L. Rubber-toughened polyurethane network and composite mateirals. Polymer 1987;28:356-67.

Dai J. B., Kuan H. C., Du X. S., Dai S. C. and Ma J. Development of a novel toughener for epoxy resins. Polym Int 2009;58:838-45.

Evans E. and Ritchie K. Dynamic strength of molecular adhesion bonds. Biophys J 1997;72(4):1541-55.

Federal Emergency Management Agency. Taking shelter from the storm: building a safe room for your home or small business. FEMA P-320. Washington DC; 2008.

Federal Emergency Management Agency. Design and construction guidance for community safe rooms. FEMA P-361. Washington DC; 2008.

Gersch W. and Sharpe D. R. Estimation of power spectra with finite-order autoregression models. IEEE Trans Automat Contr 1973;18(4):367-9.

Hu H., Yu S., Wang M., Ma J. and Liu K. Tribological properties of epoxy/ polyurea composite. Polymers for Advanced Technologies 2008; 20: 748-52.

International Code Concil \& National Storm Shelter Association. NSSA standard for the design and construction of storm shelters. ICC 500; 2008.

Kuan H. C, Dai J. B. and Ma J. A reactive polymer for toughening epoxy resin. J Appl Polym Sci 2010;115:3265-72.

Lakes R. S. High damping composite materials: effect of structural hierarchy. J Compos Mater 2002;36(3):287-97.

Nashif A. D., Jones D. I. G. and Henderson J. P. Vibration damping. New York, NY: Jonh Wiley \& Sons; 1985.

Rao S. S. Vibration of continous systems. New York, NY: John Wiley \& Sons; 2007.

Tsai J. L., Huang B. H. and Cheng Y. L. Enhancing fracture toughness of glass/epoxy composites by using rubber particles together with silica nanoparticles. J Comp Mater 2009;43(25):3107-23.

Zhou H. and Attard T. L. Rehabilitation and strength sustainability of fatigue damaged concrete-encased steel flexural members using a newly developed polymeric carbon-fiber composite. Comp Part B: Eng 2013;45:1091-103.

Zhou H., Attard T. L., Wang Y. L., Wang J. A. and Ren F. Rehabilitation of notch damaged steel beams using a carbon fiber reinforced hybrid polymeric-matrix composite. Compos Struct 2013;106:690-702.

Zhou H., Dhiradhamvit K. and Attard T. L. Tornado-borne debris impact performance of an innovative storm safe room system protected by a carbon fiber reinforced hybrid-polymer matrix composite. Engineering Structures 2014;59:308-19.

Ungar E. E. and Dietrich C. W. High-frequency vibration isolation. J Sound Vib 1966;4(2):224-41. 
